Sánchez A, Ovejero Paredes K, Ruiz-Cabello J, et al. Hybrid Decorated Core@Shell Janus Nanoparticles as a Flexible Platform for Targeted Multimodal Molecular Bioimaging of Cancer. ACS Applied Materials \& Interfaces. 2018;10(37):31032-31043. doi: 10.1021/acsami.8b10452. This article may be used for noncommercial purposes in accordance with ACS Terms and Conditions for Self-Archiving. 


\title{
Hybrid Decorated Core@Shell Janus Nanoparticles As Flexible Platform For Targeted Multimodal Molecular Bioimaging Of Cancer.
}

\author{
Alfredo Sánchez ${ }^{\dagger}$, Karina Ovejero-Paredes ${ }^{\ddagger}$, Jesús Ruiz-Cabello $^{\perp{ }^{\dagger} \uparrow}$, Paloma Martínez-Ruíz, \\ José Manuel Pingarrón $\nabla$, Reynaldo Villalonga ${ }^{*} \dagger$, Marco Filice ${ }^{*}{ }_{\perp} \neq \S$ \\ ${ }^{\dagger}$ Nanosensors \& Nanomachines Group, Department of Analytical Chemistry, Faculty of \\ Chemistry, Complutense University of Madrid, Madrid, Spain. \\ ${ }^{\ddagger}$ Fundación Centro Nacional de Investigaciones Cardiovasculares Carlos III (CNIC), Melchor \\ Fernández Almagro, 3. 28029, Madrid, Spain.
}

${ }^{\perp}$ Nanobiotechnology Group, Department of Chemistry in Pharmaceutical Sciences, Faculty of Pharmacy, Complutense University of Madrid (UCM), Plaza Ramón y Cajal, 28040 Madrid, Spain

$\S$ CIBER de Enfermedades Respiratorias (CIBERES), Melchor Fernández Almagro, 3. 28029, Madrid. Spain.

${ }^{\dagger}$ CIC biomaGUNE, 20014, Donostia-San Sebastián, Spain.

"IKERBASQUE, Basque Foundation for Science, Spain

a Department of Organic Chemistry I, Faculty of Chemistry, Complutense University of Madrid, 28040 Madrid, Spain.

$\nabla$ Department of Analytical Chemistry, Faculty of Chemistry, Complutense University of Madrid, Madrid, Spain.

*Corresponding authors: rvillalonga@quim.ucm.es (RV). mfilice@ucm.es (MF). 
Keywords: Hybrid Janus nanoparticle, multimodal molecular imaging, MRI, CT, Fluorescence Imaging, theranosis, drug delivery, nanotechnology, cancer imaging, gold nanoparticles, mesoporous silica nanoparticles. 


\begin{abstract}
In the recent years, targeted cancer theranosis -the concomitant therapeutic treatment and selective visualization of cancerous tissue- has become a powerful strategy to improve patient prognosis. In this context, targeted multimodal molecular imaging -the combination of different imaging modalities overcoming their individual limitations- has attracted great attention. Due to their unique properties, advanced nanomaterials have taken center stage in the development of theranostics. In this work, we report a novel Janus nanoplatform by combining a $\mathrm{Fe}_{3} \mathrm{O}_{4} \mathrm{NPs} /$ mesoporous silica core@shell face together with an Au nanoparticle face. Due to its anisotropy, this hybrid nanomaterial enabled the orthogonal site-selective modification of each face permitting the incorporation of a targeting peptide for cancer detection (cRGD) and a fluorescent dye. Due to the intrinsic characteristics of this Janus nanoplatform together with those selectively generated on their surfaces, the resulting hybrid nanocarrier successfully promoted the in vivo tumor-targeted multimodal imaging by magnetic resonance $\left(\mathrm{Fe}_{3} \mathrm{O}_{4}\right.$ core), computed tomography (AuNP face) and fluorescent tracking (fluorescent dye loading) in fibrosarcoma-bearing mouse model. The achieved results endorse these hybrid Janus nanoparticles as a powerful and flexible platform with integrated imaging and carrier functionalities to be equipped with therapeutic features to generate an advanced multifunctional nanocarrier for targeted cancer theranosis.
\end{abstract}




\section{Introduction}

Personalized, or sometime defined precision medicine, is a novel approach arisen in medicine (mainly in cancer treatment) whose main scope is improving patients' healthcare during the entire disease course, starting from the diagnosis up to the final follow up. ${ }^{1,2}$ In this context, theranosis (defined as the integration of therapeutic and diagnostic abilities on a single platform able to diagnose, to site-specifically carry drugs and to monitor the therapy allowing the development of personalized treatment) has received great attention in the last years. ${ }^{2,3}$ By this strategy, thanks to the careful fusion of molecular imaging together with molecular therapeutic modalities, the precise real-time tracking of the theranostic carrier and the visualization of its site-specific activity within body tissues will be achieved. As consequence, the early detection and staging of a given disease together with the most proper therapy selection and follow up can be promoted. Furthermore, due to this precise tissue accumulation visualization, the efficacy and toxicities of a specific drug could be predicted. ${ }^{4}$ The final evaluation of all these informations will allow adjusting or modifying the therapeutic strategy.

In this context, nanotechnology has played a crucial role in the development of theranostic tools. $^{5}$ In fact, nanoparticles show extremely useful characteristics to be utilized in theranostics preparation. For example, high surface-area-to-volume ratio allowing high therapeutic and imaging moieties loading, wide set of surface functionalization with ligands or reduced dimensions permitting extravasation to leaky vasculature, are only some of the most relevant nanoparticle features. ${ }^{2}$ Many elegant examples of multiactive nanostructures for simultaneous therapy and diagnosis applications have been described in literature. ${ }^{6-9}$

A promising step over in smart nanoparticle synthesis expects that they can be designed also to modulate the release of a cargo (e.g. drug) upon environmental stimuli such as $\mathrm{pH}$, temperature, enzymes or redox potential. $^{10}$ To reach this scope, Janus particles -anisotropic nanomaterials as termed by Nobel Prize de Gennes referring to double-faced 
head Roman God-, have emerged as a promising and powerful research area of colloidal structures with biomedical applications. ${ }^{11,12}$ Toward this direction, different scientific researches explored the ability of Janus nanoparticles (JNP) in multifunctional controlled drug delivery especially for cancer therapy. ${ }^{13,14}$

For example, we have recently reported the preparation of JNPs having Au and mesoporous silica opposite faces, which were properly modified with a $\mathrm{pH}$-responsive supramolecular nanovalve based on a $\beta$-cyclodextrin structure placed on the silica mesoporous surface and with two enzymatic effectors immobilized on the $\mathrm{Au}$ face. ${ }^{15}$ These smart nanodevices for stimuli-responsive release controlled by biochemical logical operator were loaded with an anticancer drug (doxorubicin) and successfully evaluated in vitro with HeLa cancer cells showing a very promising potential for cancer treatment. ${ }^{15}$

However, in comparison with this research line mainly focusing the therapeutic potential of Janus nanomaterials, only few studies explored their molecular imaging and non-invasive diagnostic potential. In fact, together with the already proven therapeutic ability, ${ }^{15}$ if this Janus platform were also equipped with targeted delivery and multimodal molecular imaging features, an enhanced smart theranostic stimuli-responsive nanosystem would be achieved. Due to the orthogonal multi-faced design of Janus nanocarriers, multiple imaging properties can be properly created, being this multimodal imaging characteristic a key element for the development of PM-targeting tool. In this sense, these anisotropic nanoparticles have the advantage of comprising two different faces enabling two complementary modification chemistries. Moreover, the presence of a porous carrier makes possible including a vast set of widely biologically active moieties.

In the past two decades, the development of probes for molecular imaging based on engineered nanomaterials has undergone to an impressive growth. The main scope of these highly promising nanoprobe classes is to enable the precise detection and quantification in 
space and over the time of physio-pathological processes finally permitting an early disease diagnosis with high sensitivity and specificity. ${ }^{16-19}$ Due to its intrinsic physic characteristics, each individual imaging modality can provide only limited information in terms of spatial and temporal resolution, sensitivity and penetration depth, being unable promoting an accurate imaging diagnosis by itself individually. ${ }^{20-22}$ In other terms, no single molecular imaging technique is able to provide a 'perfect' diagnosis. Consequently, the multimodal molecular imaging concept (combination of different imaging techniques) has rapidly become the reference strategy to bypass the intrinsic limitations of each individual imaging modality while maximizing its advantages. ${ }^{23,24}$

Within the clinically approved ones, the computed tomography (CT) promoted by X-rays is an important imaging modality whose best advantages rely in a very high spatial resolution and unlimited penetration depth. ${ }^{25,26}$ To promote this powerful imaging modality, thanks to its extremely high X-ray absorption coefficient, gold nanoparticles represent one of the best contrast agent available. ${ }^{27}$ Unfortunately, CT modality is also characterized by a low sensitivity. ${ }^{25}$ Consequently, to compensate that limitation, some imaging modalities with high sensitivity should be coupled to Computed Tomography in order to develop a complementary multimodal imaging probe.

Toward this scope, for example red to near-infrared (Red-NIR, 600-900 nm) optical imaging (OI) is a valuable strategy for non-invasive detection because of its high sensitivity and facile operability. ${ }^{28}$ Considering that in this region the absorption and auto-fluorescence of biological tissues is relatively low, fluorescent Red-NIR nanoprobes presenting long wavelength emission are the best option in order to enable the OI-based detection. ${ }^{29}$ Despite these advantages, even OI modality presents some limitations. In more details, poor spatial resolution, tissue attenuation and reduced tissue penetration make this modality very useful for in vivo qualitative tracking but not for satisfactory quantification. ${ }^{21}$ 
To compensate the above described drawbacks, among all non-invasive clinical imaging modalities, Magnetic Resonance Imaging (MRI) results as the most attractive one because of its lack of radiation, its ability to facilitate deep tissue penetration and, mainly, to provide high spatial resolution. ${ }^{22,30,31}$

Consequently, based on the considerations made above, we planned joining these three imaging modalities -CT, MRI and OI- thus developing a finely designed complementary molecular imaging agent with high spatial resolution, high sensitivity, deep tissue penetration and easy operational features (e.g. no radioactive nuclei application such as in positron emission tomography (PET) imaging). This trimodal imaging probe results easy to construct since the physical platform -the anisotropic nanoparticles- intrinsically include two contrast agents and the combination of two different faces showing complementary chemical behavior finally facilitating further modifications through orthogonal chemistry.

In this work, we describe a novel Janus nanoplatform by combining a magnetic/mesoporous silica core@shell face with a gold nanoparticle face. The intrinsic characteristics of Au and Fe oxide constituting NPs have been exploited to generate contrast in CT and MRI imaging, respectively. Furthermore, due to the different chemical reactivity of its structure, this anisotropic nanomaterial enabled the orthogonal site-selective modification of each face. Hence, the selective incorporation of a targeting peptide for cancer detection (cRGD motif) and a fluorescent dye for OI tracking has been achieved. After full chemical and physical characterization, the developed multimodal imaging nanoplatform (RAM) has been successfully assessed as contrast agent in vitro and in vivo by tumor-targeted imaging of fibrosarcoma-bearing mouse model (Scheme 1). Finally, the biodistribution pattern and toxicity over different cell lines have been evaluated. 

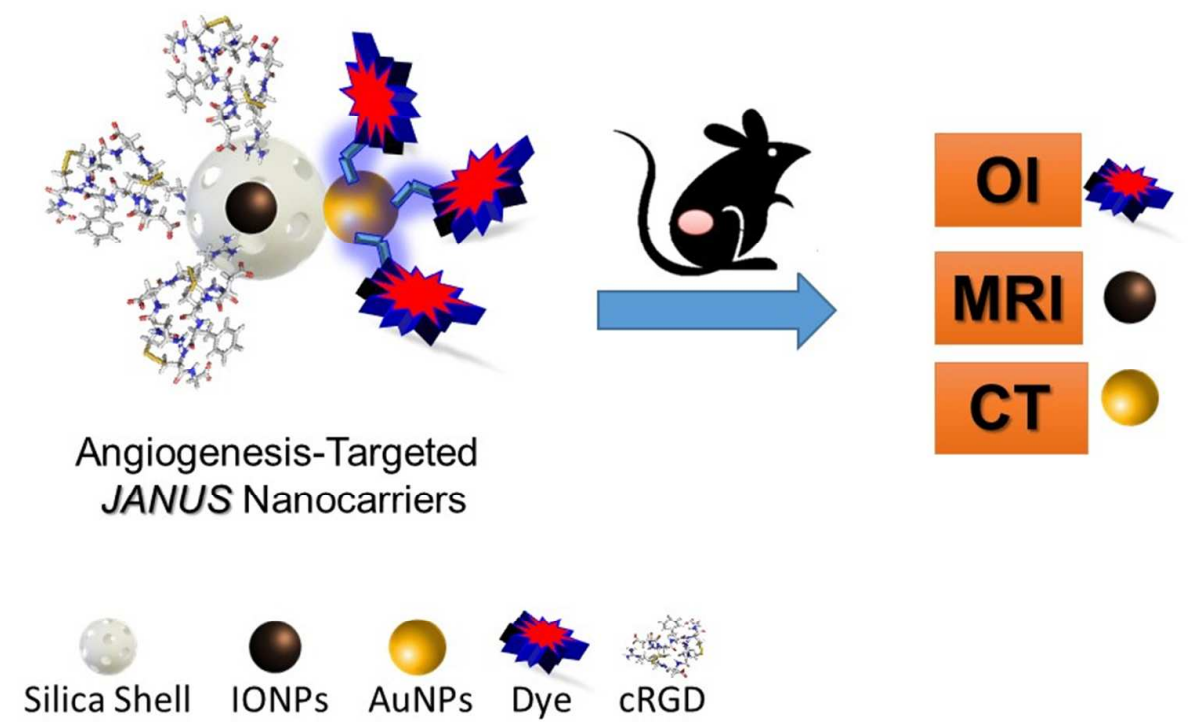

Scheme 1. General strategy for multimodal molecular imaging assessment of targeted Janus nanoparticles in tumor-bearing mouse model.

\section{Experimental Section}

\section{Materials}

Iron chloride hexahydrate, hydrogen tetrachloroaurate and cetyltrimethylammonium bromide (CTAB) were acquired from Alfa Aesar. Oleic acid (90\%), 1-octadecene (95\%) tetraethoxysilane (TEOS, 98\%), sodium citrate dihydrate, (3mercaptopropyl)trimethoxysilane, 3-(Triethoxysilyl)propyl isocyanate, paraffin wax, 3mercaptopropionic acid, N-Hydroxysuccinimide (NHS) and N-(3-Dimethylaminopropyl)-N'ethylcarbodiimide hydrochloride (EDAC) and 3-methylcholanthrene were purchased from Sigma-Aldrich. Sodium oleate (97\%) was obtained from TCI Chemicals, Alexa Fluor ${ }^{\mathrm{TM}} 647$ Hydrazide (1mg/mL) from ThermoFisher and peptide cyclo[Arg-Gly-Asp-D-Phe-Lys] (cRGD) was from ABX advanced biochemical compounds (Gmbh, Germany). Solvents were provided by Scharlau. All other reagents were of analytical grade.

\section{Animals and ethics}


Mice were housed in specific facilities (pathogen-free for mice) at the CNIC. All animal experiments were carried out after previous approval by the ethics and animal welfare committee at CNIC and in agreement to the Spanish Legislation and UE Directive $2010 / 63 / \mathrm{EU}$

\section{Methods}

Preparation of spherical magnetic nanoparticles (Mag 320). The oleic capped magnetic nanoparticles were prepared according to a 2 steps approach previously described. ${ }^{32}$ Firstly, iron-oleate complex is produced. A solution containing the iron (III) chloride precursor (2.2 $\mathrm{g}$ ) and sodium oleate $(7.4 \mathrm{~g})$ in a mixture of ethanol, water and hexane with a volume ratio of 16.3:12.2:28.5 is heated to reflux (at about $130{ }^{\circ} \mathrm{C}$ ) with magnetic stirring and kept at this temperature for $4 \mathrm{~h}$. Thereafter, the non-reacted reagents were extracted with water using a separatory funnel. Finally, the dark brown oil was dried with a rotary evaporator.

In a second step, $1 \mathrm{~g}$ of this oil, $7.1 \mathrm{~mL}$ of 1 -octadecene and $177.3 \mu \mathrm{L}$ of oleic acid were added in a two-necks round bottom flask. This mixture was dried under vacuum at $80{ }^{\circ} \mathrm{C}$ during $30 \mathrm{~min}$. Then, the mixture was heated to $320{ }^{\circ} \mathrm{C}$ using a sand bath with strong stirring and nitrogen flow. After $1 \mathrm{~h}$, the reaction was cooled to room temperature and the magnetic nanoparticles were then precipitated with ethanol, recovered with a magnet and washed several times with a mixture of hexane:ethanol (1:3). Finally, the nanoparticles were washed twice with hexane, and the solid isolated using a centrifuge.

\section{Preparation of magnetic@mesoporous silica core@shell structured nanoparticles (Mag 320@MS). ${ }^{32}$}

Initially a phase-exchange of magnetic nanoparticles was required. To do that, $20 \mathrm{mg}$ of dried oleic capped magnetic nanoparticles were sonicated in $2 \mathrm{~mL}$ of chloroform to obtain a homogeneous suspension. Then, $400 \mathrm{mg}$ of CTAB were poured into this suspension and, subsequently, $20 \mathrm{~mL}$ of ultrapure water were added into the flask under vigorous stirring. 
After that, the chloroform was removed at $60^{\circ} \mathrm{C}$ with vigorous stirring, yielding an aqueous stable solution of magnetic nanoparticles in CTAB.

Secondly, $43 \mathrm{~mL}$ of ultrapure water and $\mathrm{NaOH} 2 \mathrm{M}(350 \mu \mathrm{L})$ were stirred at $80{ }^{\circ} \mathrm{C}$ in a twonecks round bottom flask. While maintaining this temperature, $5 \mathrm{~mL}$ of the CTAB-magnetic nanoparticles -which were passed through a syringe filter $(0.45 \mu \mathrm{m})$ to eliminate agglomerates- were dropped to the basic solution. Finally, $0.5 \mathrm{~mL}$ of TEOS were dropped slowly, and the reaction was stirred for $2 \mathrm{~h}$.

The solution was cooled and the magnetic solid was purified using a magnet and rinsed several times with water. Finally, the CTAB from the pores was removed by heating the solid at $60{ }^{\circ} \mathrm{C}$ for $30 \mathrm{~min}$ in $60 \mathrm{~mL}$ of ethanol with $160 \mathrm{mg}$ of $\mathrm{NH}_{4} \mathrm{NO}_{3}$. Thereafter, the solid was washed with ethanol (3x) and dried at room temperature.

Preparation of 18-nm Au nanoparticles. According to Frens's method, ${ }^{33} 50 \mathrm{~mL}$ of a recently prepared $0.01 \% \mathrm{w} / \mathrm{w}$ solution of $\mathrm{HAuCl}_{4}$ was boiled and then, $750 \mu \mathrm{L}$ of a $1 \% \mathrm{w} / \mathrm{w}$ solution of trisodium citrate were suddenly injected. The mixture was allowed boiling for 10 min, cooled and then, the volume was adjusted with ultrapure water to the original value, finally obtaining a stable colloidal solution.

\section{Preparation of Janus Au-magnetic@MS nanoparticles (Janus Au-Mag 320@MS).}

Janus nanoparticles have been prepared as previously described by our group. ${ }^{15,34}$ Briefly, 200 mg of Mag 320@MS nanoparticles were sonicated in a CTAB $1.0 \mu \mathrm{M}$ solution in ethanolwater $(6.7 \% \mathrm{v} / \mathrm{v}, 10 \mathrm{~mL})$. After heating the suspension at $75{ }^{\circ} \mathrm{C}, 1 \mathrm{~g}$ of paraffin wax was poured to the mixture and left melting. After that, the mixture was emulsified with an UltraTurrax T-18 homogenizer (IKA, Germany) for $5 \mathrm{~min}$ at $25000 \mathrm{rpm}$. The obtained emulsion was further stirred whit a magnetic bar for $1 \mathrm{~h}$ at $1000 \mathrm{rpm}$ at the same temperature $\left(75^{\circ} \mathrm{C}\right)$ finally yielding a Pickering emulsion. This emulsion was then cooled to room temperature, 
diluted with methanol and allowed reacting for $3 \mathrm{~h}$ with (3-mercaptopropyl) trimethoxysilane $(200 \mu \mathrm{L})$. Thereafter, the functionalized solid was recovered by filtration and the excess of silane was removed with methanol and then, the solid was dispersed in $400 \mathrm{~mL}$ of $18 \mathrm{~nm} \mathrm{Au}$ nanoparticles solution. The suspension was left reacting overnight with magnetic stirring; after filtration, the solid was rinsed with ultrapure water. After that, the solid was washed several times with methanol using a centrifuge and finally chloroform was employed to remove the paraffin wax.

\section{Preparation of the cRGD-targeted and fluorescent Janus nanoparticles (RAM).}

To modify selectively the gold face of the prepared Janus nanoparticles with a fluorescent molecule, the Janus Au-Mag 320@MS nanoparticles (15 mg) were suspended in $4 \mathrm{~mL}$ of methanol and allowed reacting for $1 \mathrm{~h}$ with 3-mercaptopropionic acid. The nanoparticles were magnetically precipitated with a neodymium permanent magnet and rinsed twice with methanol and two more times with cold $50 \mathrm{mM}$ phosphate buffer, $\mathrm{pH} 4.5$. Then, the nanoparticles were suspended in the same buffer and $2 \mathrm{mg}$ of NHS, $2 \mathrm{mg}$ of EDAC and $75 \mu \mathrm{L}$ of Alexa Fluor 647 Hydrazide were poured with the functionalized nanoparticles. These reagents were kept overnight under stirring in an ice bath. Subsequently, the fluorescent nanoparticles were separated by centrifugation and rinsed twice with the same cold buffer and two other times with methanol.

For the silica site-selective modification, the resulting nanoparticles were dispersed in $4 \mathrm{~mL}$ of methanol and $20 \mu \mathrm{L}$ of 3-(Triethoxysilyl)propyl isocyanate were dropped into the solution. After $3 \mathrm{~h}$ stirring, the solid was collected and rinsed with methanol and twice with cold 50 $\mathrm{mM}$ phosphate buffer, $\mathrm{pH}$ 7. Thereafter, the nanoparticles were suspended in $4 \mathrm{~mL}$ of the same buffer and left to react with $1 \mathrm{mg}$ of cRGD peptide overnight at $4^{\circ} \mathrm{C}$. Finally, the targeted Janus nanoparticles were washed and diluted to $1 \mathrm{mg} / \mathrm{mL}$ with a sterile saline solution $(\mathrm{NaCl} 0.9 \% \mathrm{w} / \mathrm{w})$. 


\section{Physicochemical characterization of nanomaterials.}

Morphology and size of Janus nanocarriers were determined by transmission electron microscopy (TEM) bright field measurements performed with a JEOL JEM-2100 microscope. HAADF-STEM images and EDX mapping were acquired using a JEOL JEM 3000F microscope equipped with a XEDS detector (OXFORD INCA). Spectrophotometric measurements were performed using an Ultrospec ${ }^{\mathrm{TM}} 8000$ Dual Beam UV/VIS spectrophotometer $\left(\right.$ Biochrom $\left.^{\mathrm{TM}}, \mathrm{UK}\right)$. The fluorescent spectra from the nanoparticles were acquired with a Cary Eclipse Spectrophotometer (Agilent, Santa Clara, USA). Powder X-ray diffractograms were collected with an X'Pert MRD diffractometer (PANanalytical B.V., The Netherlands). An ASAP 2020 Physisorption Analyzer (Micromeritics, USA) was used to obtain the gas adsorption/desorption isotherms and pore size distributions. Thermogravimetric analysis were carried out with an SDT-Q600 (TA Instruments, USA). FT-IR spectra were recorded on a Perkin Elmer Spectrum 400 Series spectrometer (Perkin Elmer, USA); Inductively coupled plasma atomic emission spectrometry (ICP-AES) was performed on a Perkin Elmer OPTIMA 2100 DV equipment. A Zetasizer Nano ZS90 (Malvern Instruments, UK) with folded capillary cells was employed for analysing the hydrodynamic size, polydispersity index and zeta potential of all the NPs.

\section{Magnetic characterization}

Magnetic characterization was carried out in a vibrating sample SQUID Magnetometer (Quantum Design, model MPMS-XL, USA). To prepare the samples, $100 \mu \mathrm{L}$ of aqueous particle suspension were dropped to cotton wool piece. The particle loaded cotton support was let drying at room temperature overnight and then it was inserted into a gelatine capsule. The field-dependent magnetization measurements were carried out at $298 \mathrm{~K}$ up to $5 \mathrm{~T}$.

To determine longitudinal and transverse relaxation times, five different concentrations of the nanoparticle suspension were analysed in a Bruker Minispec (Bruker BioSpin GmbH, 
Germany) mq60 relaxometer at $1.5 \mathrm{~T}$ and $37^{\circ} \mathrm{C}$. The standard Carr-Purcell-Meiboom-Gill multi spin echo and Inversion Recovery spin echo sequences were applied to determine $T_{2}$ and $T_{1}$ respectively. The $R_{1}$ and $R_{2}$ values were achieved by plotting the retrieved relaxation values against the Fe concentration in $\mathrm{mM}$. The slopes from the linear representation of the data correspond with the relaxivities $\left(\mathrm{r}_{\mathrm{i}}, \mathrm{s}^{-1} \mathrm{mM}^{-1}\right)$ associated with the iron concentration. $(\mathrm{mM}): \mathrm{R}_{\mathrm{i}}=\mathrm{r}_{\mathrm{i} 0}+\mathrm{r}_{\mathrm{i}}[\mathrm{Fe}]$.

\section{In Vitro Cytotoxicity of RAM}

The cells used for the cytotoxicity assay were grown and maintained as described in reference 9. After culturing all these cells, the potential toxicity arising from the administration of RAM was determined by MTT assay. Cells $\left(10^{4}\right.$ cells/well $)$ were cultured into 96-well microliter plates for $24 \mathrm{~h}$ in $5 \% \mathrm{CO}_{2}$ atmosphere. The Janus nanocarriers suspension was added at different concentrations ranging from 35 to $350 \mu \mathrm{g} / \mathrm{mL}$ in each cell-containing well. After $24 \mathrm{~h}$ incubation, the medium was replaced with fresh medium containing MTT $(0.5 \mathrm{mg} / \mathrm{mL}, 100$ $\mu \mathrm{L})$ at $37^{\circ} \mathrm{C}$. After $4 \mathrm{~h}$ incubation, the MTT medium was replaced with $100 \mu \mathrm{L}$ DMSO and incubated for $10 \mathrm{~min}$ at $37^{\circ} \mathrm{C}$ in order to dissolve the formazan salt. The cell viability was determined by measuring the absorbance of each well at $540 \mathrm{~nm}$ using a Bio-Rad 680 microplate reader. Untreated cells were used as control. All experiments were performed in triplicate.

\section{Fibrosarcoma-bearing mouse model}

The 3-methylcholanthrene-induced fibrosarcoma was generated as extensively reported in literature ${ }^{35}$ using 8-12-weeks old C57BL/6J male mice. Animals were housed at $22{ }^{\circ} \mathrm{C}$, under 12h light/dark cycle with freely available water and food. The fibrosarcoma was generally generated within the next 90-120 days post intramuscular injection.

\section{In vitro and in vivo assessment of imaging potential of RAM by Magnetic Resonance.}


In vitro and in vivo MRI was performed with an Agilent/Varian scanner (Agilent, Santa Clara, USA) controlled by a DD2 console and active-shielded by 205/120 gradient insert coil with $130 \mathrm{mT} / \mathrm{m}$ maximum gradient strength and a combination of a volume coil and a 2-channel phased-array (Rapid Biomedical GmbH, Rimpar, Germany).

In vitro assessment of MRI of RAM samples was carried out using a standard spin echo sequence under the following conditions: Repetition Time (TR): 2500ms; Echo Time (TE): 10ms; Echo Number (NE): 16; Average: 3; Field Of View (FOV): 25 x 25 mm; Matrix: 128 x 128.

For In vivo MRI, male C57BL/6 fibrosarcoma bearing mice $(\mathrm{n}=3)$ were anesthetized with $2 \%$ isoflurane (Abbott) and oxygen, and positioned on a mouse bed with constant monitoring of respiratory cycle (SA Instruments, NY). In order to prevent retinal drying, ophthalmic gel was used. Baseline images were acquired before intravenous (i.v.) administration of the probes $(100 \mu \mathrm{L}$ at $1 \mathrm{mg} \mathrm{Fe} / \mathrm{mL})$. MRI data were acquired $1 \mathrm{~h}, 2 \mathrm{~h}$ and $3 \mathrm{~h}$ after injection. Axial images were acquired in free-breathing animals, using a gradient echo 3D sequence with echo (TE)/repetition time (TR): $1.65 \mathrm{~ms} / 3 \mathrm{~ms}$; FOV: 27.8 × 30.33 × $26.44 \mathrm{~mm}$; averages: 5; flip angle of $20^{\circ}$ and a matrix: $256 \times 256 \times 256$.

\section{In vivo Fluorescence Imaging and biodistribution pattern analysis.}

Fluorescence in vivo imaging was performed with IVIS Imaging System 200 series (Xenogen ${ }^{\circledR}$ ) (acquisition parameters: Cy5.5 ex/em filter, high level, BIN-HR, FOV: 6.6, f8). Data were quantified as radiant efficiency.

For biodistribution pattern evaluation of intravenously injected RAM, tumor tissues and organs including heart, liver, spleen, lung, kidney of tumor mice models $(n=3)$ treated with RAM were collected after $2 \mathrm{~h}$ p.i. and analyzed by IVIS at the same conditions reported above. The excised tumoral masses and organs from control tumour mice models without RAM 
treatment were used as control. The corrected total fluorescence intensity analysis was carried out using ImageJ.

\section{In vivo Computed Tomography imaging}

In vivo $\mathrm{CT}$ Imaging in tumor bearing mouse was performed with a nanoPET/CT small-animal imaging system (Mediso Medical Imaging Systems, Budapest, Hungary). Whole-body standard CT scans were obtained with the X-ray tube setup at a voltage of $80 \mathrm{kV}$ and current of $500 \mu \mathrm{A}$. Acquisition and reconstruction were performed with proprietary Nucline software (Mediso, Budapest, Hungary). Qualitative Image analysis in mice was performed using Osirix software (Pixmeo, Switzerland).

The Hounsfield units (HU) of RAM at different gold concentrations (25, 50, 250, 500, $\mu \mathrm{g} / \mathrm{mL}$ ) were determined using the same nanoPET/CT small-animal imaging system and the resulting images were analyzed using Osirix software. The HUs for each concentration was determined by the following equation $\mathbf{1}$ :

$$
\mathrm{HU}=\mathrm{X}^{*} \text { Rescale Slope }+ \text { Rescale Intercept (1) }
$$

(X: CT value for each Au concentration of RAM, $\mu \mathrm{g} / \mathrm{mL}$ ) being Rescale Slope and Rescale Intercept values 1 and 0 respectively. 


\section{Results and discussion}

Synthesis and characterization of Janus Au-Mag 320@MS nanoparticles.

All the reported synthetic steps carried out during the Janus nanoparticles assembly were extensively analyzed and characterized using standard techniques. As first step, the oleic capped magnetic nanoparticles were synthetized. The selection of this class of nanoparticles is not casual because the presence of oleic-capping coating will facilitate the dispersion of the nanoparticles being this last a key parameter to obtain small and homogeneous core@shell nanoparticles. After their synthesis, the oleic capped magnetic nanoparticles were analyzed by TEM and HRTEM to confirm the spherical shape morphology and analyze the average size this being $15.5 \pm 2.2 \mathrm{~nm}$ diameter (Figure 1a and Figure S1).

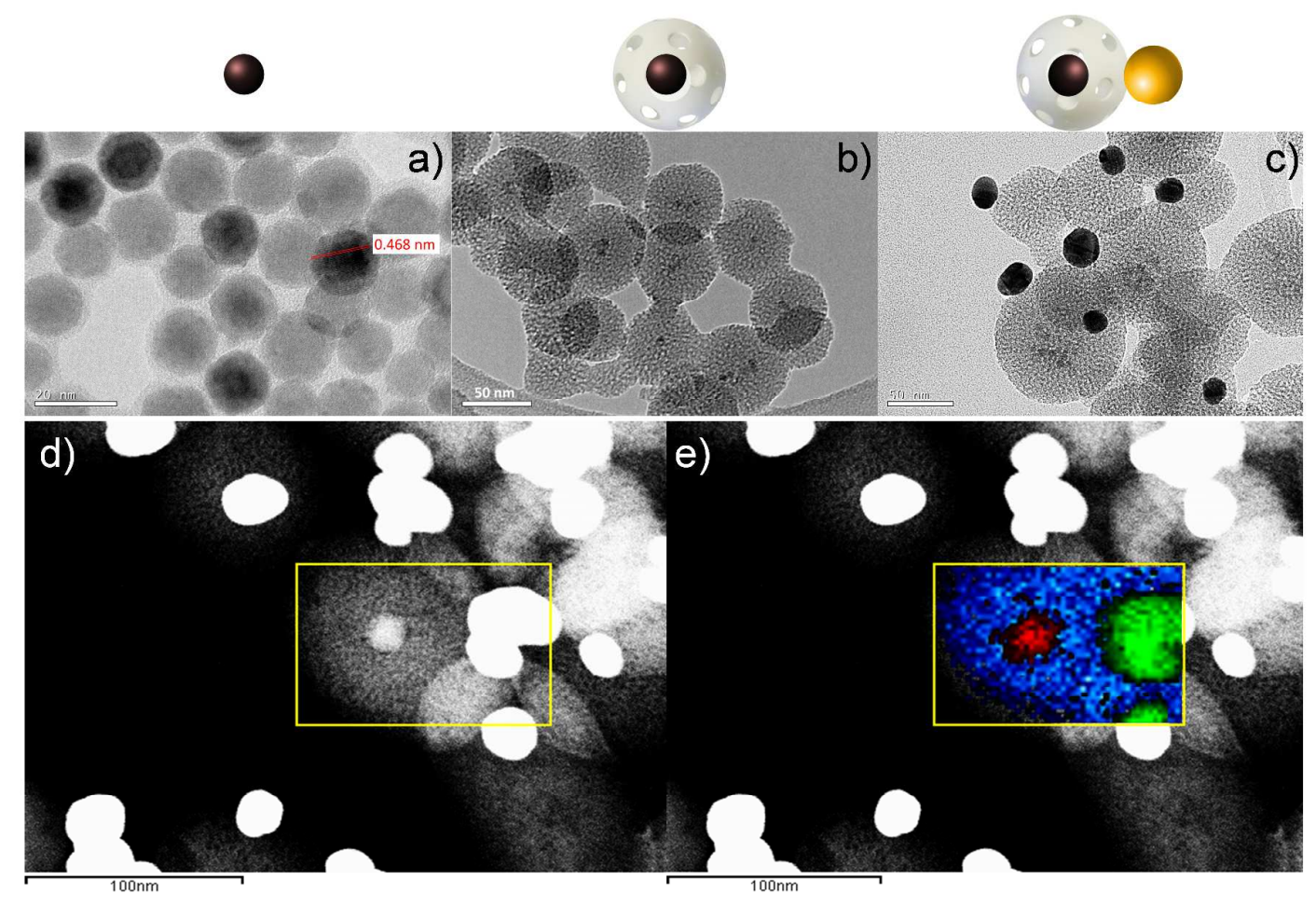

Figure 1. TEM analysis of Mag 320 (a), Mag 320@MS (b), and Janus Au-Mag 320@MS nanoparticles (c); HAADF-STEM analysis of Janus Au-Mag 320@MS nanoparticles (d); Combination of HAADF-STEM analysis and EDX mapping of constituent elements of Janus Au-Mag 320@MS nanoparticles (e). Blue: Si atoms, Red: Fe atoms and Green; Au atoms. 
The interplanar distance value measured with HRTEM image retrieved a value of $0.468 \mathrm{~nm}$ approximately which correspond to (111) planes of the $\mathrm{Fe}_{3} \mathrm{O}_{4}$ single crystal with spinel structure (Figure 1a and Figure S2). ${ }^{36,37}$ The as prepared nanoparticles were then coated with a mesoporous siliceous layer by means of silanization/porogenic agent extraction strategy. ${ }^{32}$ Thereafter, the resulting core@shell NPs were selectively modified with controlled number of AuNPs by a Pickering emulsion strategy. ${ }^{15,34}$ The preparation of the core@shell Mag 320@MS nanoparticles and the Janus Au-Mag 320@MS nanoparticles were confirmed by TEM analysis. Figure 1b and S3a show Mag 320@MS nanoparticles. Their nanostructure composed of magnetic core embedded into mesoporous silica shell can be clearly observed. The silica coating exhibited a worm-like porous distribution. The small size of these nanoparticles, $60 \pm 6 \mathrm{~nm}$, is due to the low agglomeration of the magnetic nanoparticles in the core indicating a good dispersion of the oleic capped nanoparticles after phase exchange, previously to the silica condensation. The TEM micrograph of Janus Au-Mag 320@MS nanoparticles showed clearly the three components of these anisotropic nanoparticles of about $80 \mathrm{~nm}$ overall size (Figure $1 \mathrm{c}$ and $\mathrm{S} 3 \mathrm{~b}$ ). To further confirm the Janus character of our nanoparticles, the HAADF-STEM analysis was carried out (Figure 1d). In HAADF-STEM mode, the integration of average atomic number $\mathrm{Z}$ over the sample thickness determines the image intensity. Hence, in order of decreasing $Z$, Au regions $(Z=79)$ appear with a higher intensity than Fe-rich regions $(Z=26)$ and Silicon areas $(Z=14)$ as indicated in Figure $1 \mathrm{~d}$. After that, EDX chemical mapping was performed and overlapped to HAADF-STEM image with the aim to confirm the previous observation and to deeply analyze the $3 \mathrm{D}$ distribution of the elements (Figure 1e). The achieved image confirms undoubtedly the Janus character of our nanocarrier constituted by a Fe core embedded in a silica shell that is finally decorated with gold nanoparticles (Figure 1). 
To confirm the crystalline phase of both magnetic and gold nanoparticles, as well as the mesoporous structure of silica, the nanomaterials were analyzed by XRD in the $1.8-80$ degrees $2 \theta$ range (Figure 2).

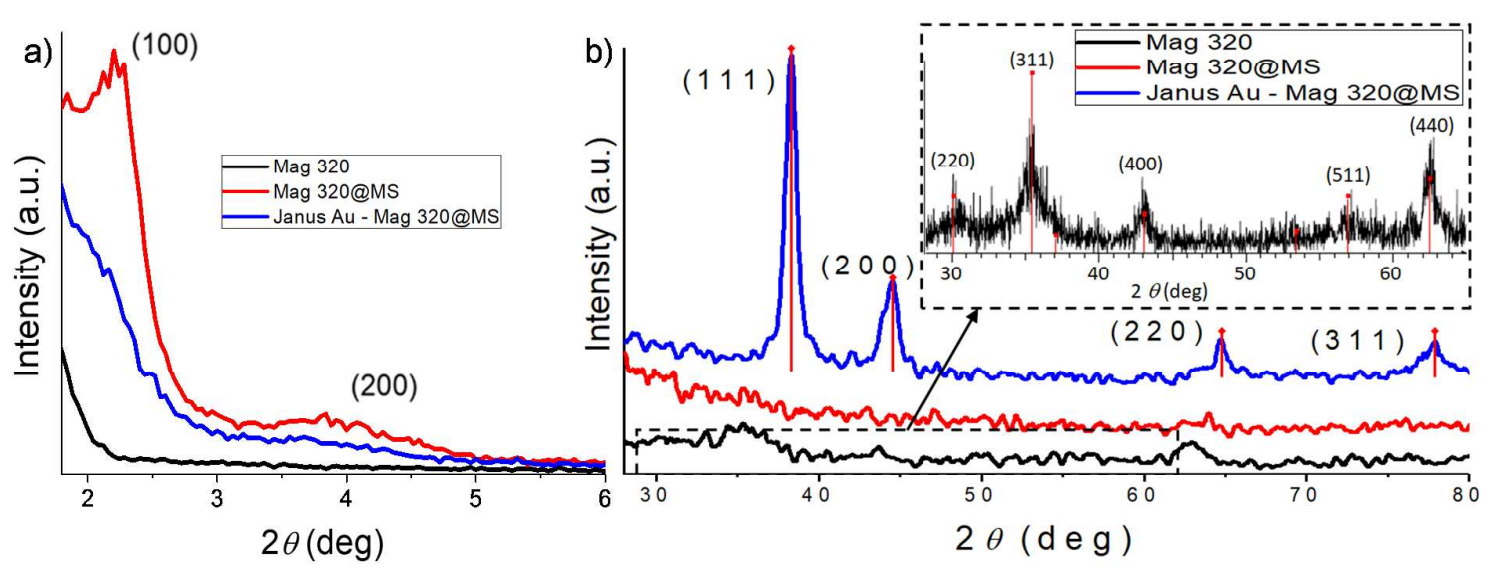

Figure 2. Powder X-ray diffraction pattern of magnetic cores, Mag 320@MS and Janus AuMag 320@MS nanoparticles at low (a) and high (b) $2 \theta$ values. Red sticks in Figure 2b indicate the standard reference peaks deposited in the Powder Diffraction Databases (PDF) identifying crystalline structure of $\mathrm{Fe}_{3} \mathrm{O}_{4}$ NPs [JCPDS Card No. PDF \#00-019-0629] and AuNPs [JCPDS Card No. PDF \#00-001-1172]

At low values of $2 \theta$, a sharp peak at $2 \theta=2.2^{\circ}$ and a faint intensity peak at about $4^{\circ}$ appeared in the Mag 320@MS nanoparticles (Figure 2a). These peaks indicated the presence of pores with uniform diameter in the mesoporous size with a non-symmetrical structure. In addition, these peaks could be related with small size particles and radial or worm-like mesoporous structures, confirming the TEM analysis. These peaks appeared attenuated in the Janus nanoparticles, for which diffractogram the (100) peak was shown as a smooth shoulder. ${ }^{38,39}$ The diffraction pattern at high angles for the starting magnetic cores showed a series of characteristic peaks typical for the inverse cubic spinel phase of $\mathrm{Fe}_{3} \mathrm{O}_{4}$, confirming the preparation of crystalline magnetite nanoparticles with a spherical morphology (Figure 2b, insert). ${ }^{40}$ After their inclusion into the mesoporous silica shell, the characteristic diffraction 
peaks were weakened and not significant signals were obtained. On the contrary, sharp diffraction signals were obtained for the Janus particles. This diffraction pattern is typical for crystalline Au nanoparticles and evidenced the formation of Janus nanoparticles (Figure $2 b$ ). The retrieved experimental diffractograms are in agreement with standard references deposited in the Powder Diffraction Databases (PDF) identifying crystalline structures of $\mathrm{Fe}_{3} \mathrm{O}_{4}$ NPs [JCPDS Card No. PDF \#00-019-0629] and AuNPs [JCPDS Card No. PDF \#00001-1172] (Figure 2b). After demonstrating the correct preparation of the nanomaterials, the Janus Au-Mag 320@MS nanoparticles were further characterized to evaluate their properties for the proposed biomedical application. Thus, the mesoporous nanomaterials were characterized by $\mathrm{N}_{2}$ adsorption-desorption isotherms, evaluated by BET analysis (Figure 3).
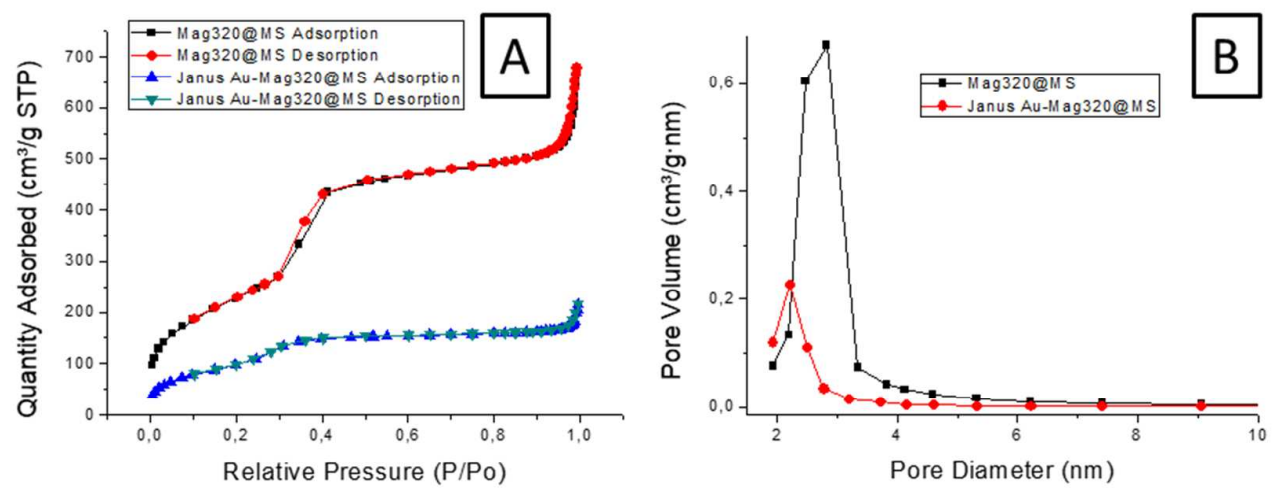

Figure 3. Nitrogen adsorption/desorption isotherms (a) and pore size distribution (b) for Mag 320@MS and Au-Mag 320@MS JNPs.

The results obtained for Mag 320@MS and Au-Mag 320@MS JNPs showed a similar behavior, with lower values of $\mathrm{N}_{2}$ adsorption quantity in the Janus nanoparticles due to the high specific weight of the gold in these nanoparticles. The isotherms corresponded with type IV according to the IUPAC classification typical of mesoporous solids. ${ }^{41}$ In this sense, a remarkable increment of the quantity adsorbed in the $\mathrm{P} / \mathrm{P}_{0}$ range of $0.25-0.4$ approximately could be ascribed to the $\mathrm{N}_{2}$ condensation by capillarity in the pore. Moreover, the absence of a hysteresis loop in this range as well as the cramped pore size distribution confirmed the 
uniformity of the generated pores, with a diameter of $2.8 \mathrm{~nm}$ for the Mag 320@MS nanoparticles and $2.2 \mathrm{~nm}$ in the Janus Au-Mag 320@MS nanoparticles. It is worth of note as these pore size values are compatible with the entrance of a therapeutic payload such as an anticancer drug (e.g. doxorubicin). ${ }^{15}$ Another notable increase in the adsorbed quantity appeared in the isotherms for $\mathrm{P} / \mathrm{P}_{0}>0.9$, which is related to the condensation of nitrogen into the porous at the nanoparticles. The specific surface for Mag 320@MS nanoparticles from the BET model was $848 \mathrm{~m}^{2} / \mathrm{g}$ and a decrease to $380 \mathrm{~m}^{2} / \mathrm{g}$ was measured after the incorporation of gold nanoparticles to yield the anisotropic nanomaterial (Figure 3). This strong decrease could be related to partial masking of the porous silica surface by the attached gold nanoparticles. After the physical characterization, the amount of Fe and Au constituting the Janus NPs was quantified by inductively coupled plasma atomic emission spectroscopy (ICP-AES). The obtained values were 0.04 and $0.247 \mathrm{mg} / \mathrm{mg}_{\text {Janus }}$ for $\mathrm{Fe}$ and $\mathrm{Au}$ respectively (Table $\mathrm{S} 1$ ). The prepared nanomaterials were also characterized by FTIR (Figure S4). Typical signals related to the oleic acid layer capping the magnetic nanoparticles, including narrow bands at 2920 $\mathrm{cm}^{-1}$ and $2851 \mathrm{~cm}^{-1}$ which correspond to $\mathrm{CH}_{2}$ stretching modes, and other bands in the 1300 $1700 \mathrm{~cm}^{-1}$ region related with the metal carboxilated coordination bound, ${ }^{42}$ appeared in the spectra of the Mag 320 nanoparticles. These bands were observed after coating the magnetic nanoparticles with the mesoporous silica shell, as well as the typical bands of silica materials, a broad band at $3400 \mathrm{~cm}^{-1}$ attributed to $\mathrm{O}-\mathrm{H}$ stretching of the surface silanol groups and the remaining adsorbed water molecules, and a strong peak at $1100 \mathrm{~cm}^{-1}$ from the siloxane $(-\mathrm{Si}-$ $\mathrm{O}-\mathrm{Si}-$ ) bounds, and other band at $900 \mathrm{~cm}^{-1}$ due to $\mathrm{Si}-\mathrm{O}$ bond stretching. ${ }^{15,34}$

\section{Face-selective surface modification of Janus nanoparticles.}

The results described above make the prepared Janus nanoparticles suitable potential reservoirs for the preparation of stimuli-controlled drug delivery systems. To characterize 
their biomedical potential also for in vivo site-specific multimodal imaging of cancer, we designed an orthogonal modification strategy targeting each face of the Janus nanoparticles.

In fact, one of the most relevant advantage of our straightforward approach relies in the easiness of tailored surface biofunctionalization, as in the case of a cancer targeting ligand or fluorescent dye loading. In the latter case, to modify selectively the gold face of the Janus nanoparticles with a fluorescent molecule, an Alexa Fluor 647 probe was covalently linked via EDC-mediated amidation reaction with carboxylic reactive groups previously generated by selective sulfur-gold reaction ${ }^{43}$ with mercaptopropionic acid (Scheme 2). The successful linking of dye on aureus face of Janus nanocarrier was confirmed by fluorescence spectroscopy (Figure S5).

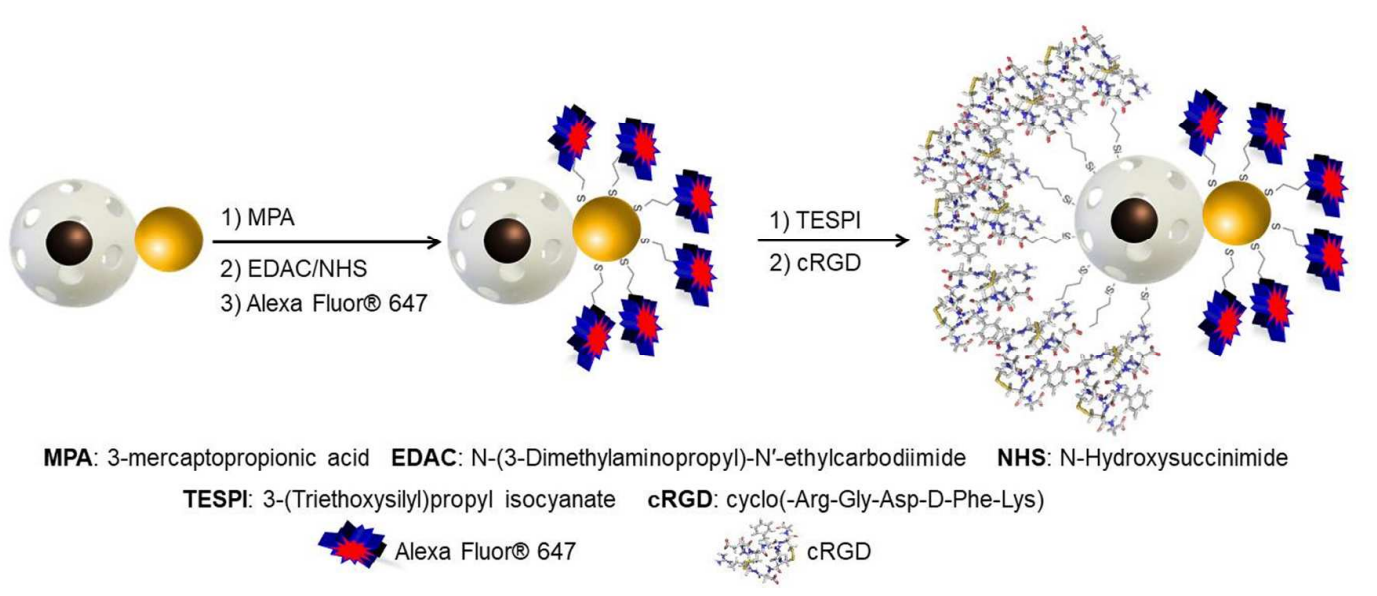

Scheme 2. Orthogonal face-selective modification of Janus nanoparticles.

On the other hand, it is well known that the arginine-glycine-aspartic acid peptide motif (RGD) specifically recognizes the $\alpha v \beta_{3}$ and $\alpha v \beta_{5}$ integrins that are surface proteins overexpressed by nascent endothelial cells during tumor angiogenesis (as well as in fibrosarcoma) but not by quiescent endothelial cells. ${ }^{44}$ This characteristic has been widely used for the successful targeting and detection of a wide range of tumor and angiogenesis processes by using a lot of probes such as chelators, polymeric nanoparticles, iron oxide nanoparticles (IONP) or quantum dots. ${ }^{45}$ Thus, a cyclic RGD motif modified with an aliphatic 
amine residue belonging to a lysine amino acid was covalently linked on the siliceous side of Janus NPs via isocyanate reactive groups previously installed by means of a selective silanization reaction (Scheme 2). The successful cRGD immobilization was checked by FTIR. As it is shown in Figure S4, the FTIR spectrum for RAM clearly shows the strong reduction of the broad band at $3400 \mathrm{~cm}^{-1}$ (the $\mathrm{O}-\mathrm{H}$ stretching of the surface silanol groups) due to the isocyanate silane modification and the typical bands related to the incorporation of the peptide: the broad band at $1635 \mathrm{~cm}^{-1}$ due to the peptide bond and the Arg side chain; the 1404 $\mathrm{cm}^{-1}$ band due to the Lys side chain. ${ }^{46}$

The surface functionalization degree of as prepared RAM Janus nanoparticles was obtained by spectrophotometric quantification of supernatant after each immobilization reaction. The functionalization degrees were 1.6 and $66 \mathrm{nmol} / \mathrm{mg}_{\mathrm{Janus}}$ for the fluorescent molecule and for the peptide, respectively. Finally, the hydrodynamic size and $\zeta$-potential of RAM nanoparticles were characterized by Dynamic Light Scattering (DLS) retrieving $163 \pm 2.8 \mathrm{~nm}$ and $-28 \pm 3.9 \mathrm{mV}$ values, respectively (Table $\mathrm{S} 1)$.

\section{In vitro assessment of multimodal imaging potential of RAM.}

Our proposed modular approach is valid for Janus nanoparticles with different designs, independently of their nature and physicochemical properties. In fact, the imaging functions of each preparation are strictly related to the inner magnetic core, the outer AuNPs decorating the Janus complex and the surface modification with fluorescent dye. Thus, we evaluated the overall potential of the JNPs as contrast agents for multimodal molecular imaging techniques in vitro using phantom of different concentration as well as in vivo animal model.

Toward this scope, the magnetic properties of RAM nanoparticles were firstly analyzed by assessing their potential as contrast agent for MRI. By vibrating sample magnetometry (VSM), the specific magnetization vs applied magnetic field values at room temperature for 
the starting magnetic cores as well as for final RAM were quantified. The results are shown in Figure 4a.
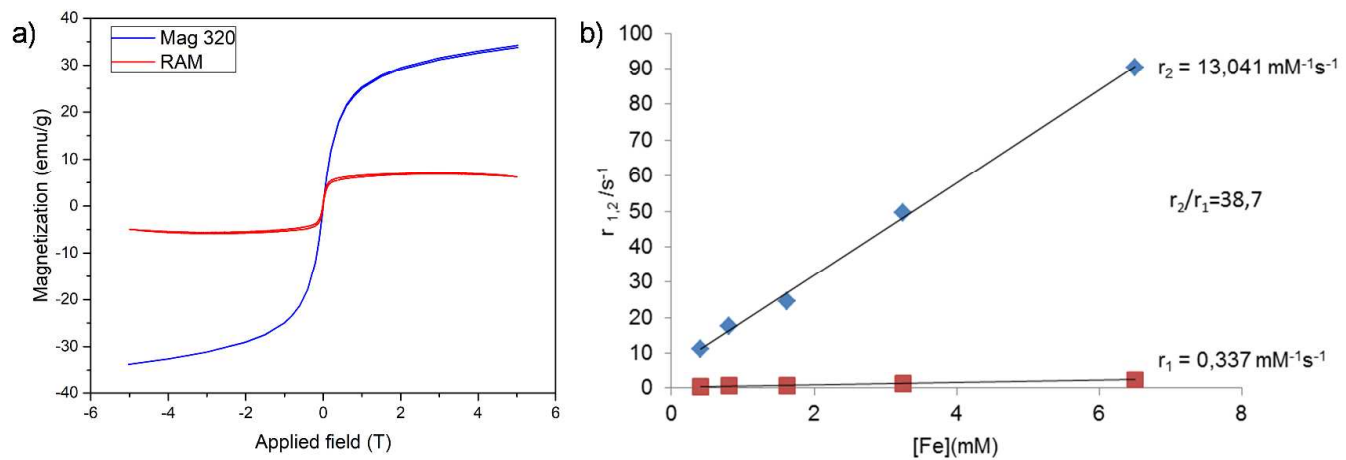

Figure 4. Magnetic properties characterization. a) Magnetization curves of starting Mag 320 and final RAM nanoparticles. b) Plots of $\mathrm{T}_{1}$ and $\mathrm{T}_{2}$ relaxation rates against iron concentration for RAM.

The magnetization curves did not show any hysteresis loop, demonstrating the superparamagnetic character of the nanoparticles. ${ }^{47}$ This property is very important for biomedical applications because its ability to prevent the nanoparticles clustering after their injection in biological environment. The saturation magnetization value of the oleic capped magnetic nanoparticles was $33.7 \mathrm{emu} / \mathrm{g}$ at $298 \mathrm{~K}$. However, after silica coating, AuNPs decoration and surface modification, the magnetization suffered a decrease up to a value of almost $7 \mathrm{emu} / \mathrm{g}$ for RAM. After that, the relaxivity properties, a crucial parameter in MRI imaging, were analyzed. In general, IONPs core confers superparamagnetic behavior and normally transversal relaxivity $\left(\mathrm{r}_{2}\right)$ for $\mathrm{T}_{2}$-weighted MRI. ${ }^{48}$ However, as demonstrated by VSM analysis, the presence of silica shell reduced the interaction of water molecules with iron core resulting in an expected $\mathrm{T}_{2}$-value reduction. Effectively, a quite low transversal $\mathrm{r}_{2}$ value of $13 \mathrm{~s}^{-1} \mathrm{mM}^{-1}$ was observed. The longitudinal $r_{1}$ value resulted very low $\left(r_{1}=0.3\right.$ $\mathrm{s}^{-1} \mathrm{mM}^{-1}$ ) with a final $\mathrm{r}_{2} / \mathrm{r}_{1}$ ratio of almost 39 (Figure $4 \mathrm{~b}$ ). This ratio value is still reasonably good in comparison with other magnetic nanoparticles used as contrast in MRI and reported in literature. ${ }^{49}$ For example, the Combidex (Guerbet), Resovist (Schering) and Endorem 
(Ferropharm) magnetic nanoparticles showed $\mathrm{a}_{2} / \mathrm{r}_{1}$ ratio of 7,9 and 12 respectively. On the other hand, for example custom-made manganese-zinc doped ferrite NPs (described to significantly enhance MRI contrast effect with respect to conventional IONPs ${ }^{50,51}$ ) coated with citric acid or amine-PEG showed a $\mathrm{r}_{2} / \mathrm{r}_{1}$ ratio of 33 and 26.5 respectively. ${ }^{49}$ This evidence together with the expected accumulation of RAM on tumor site due to the cRGD targeting moiety make these particles as a potential contrast agent for $\mathrm{T}_{2}$-weighted MRI. Indeed, this feature was successfully evaluated by MRI of phantoms with a series of dilutions in a spin echo $\mathrm{T}_{2}$-weighted sequence (Figure $5 \mathrm{a}$ ).

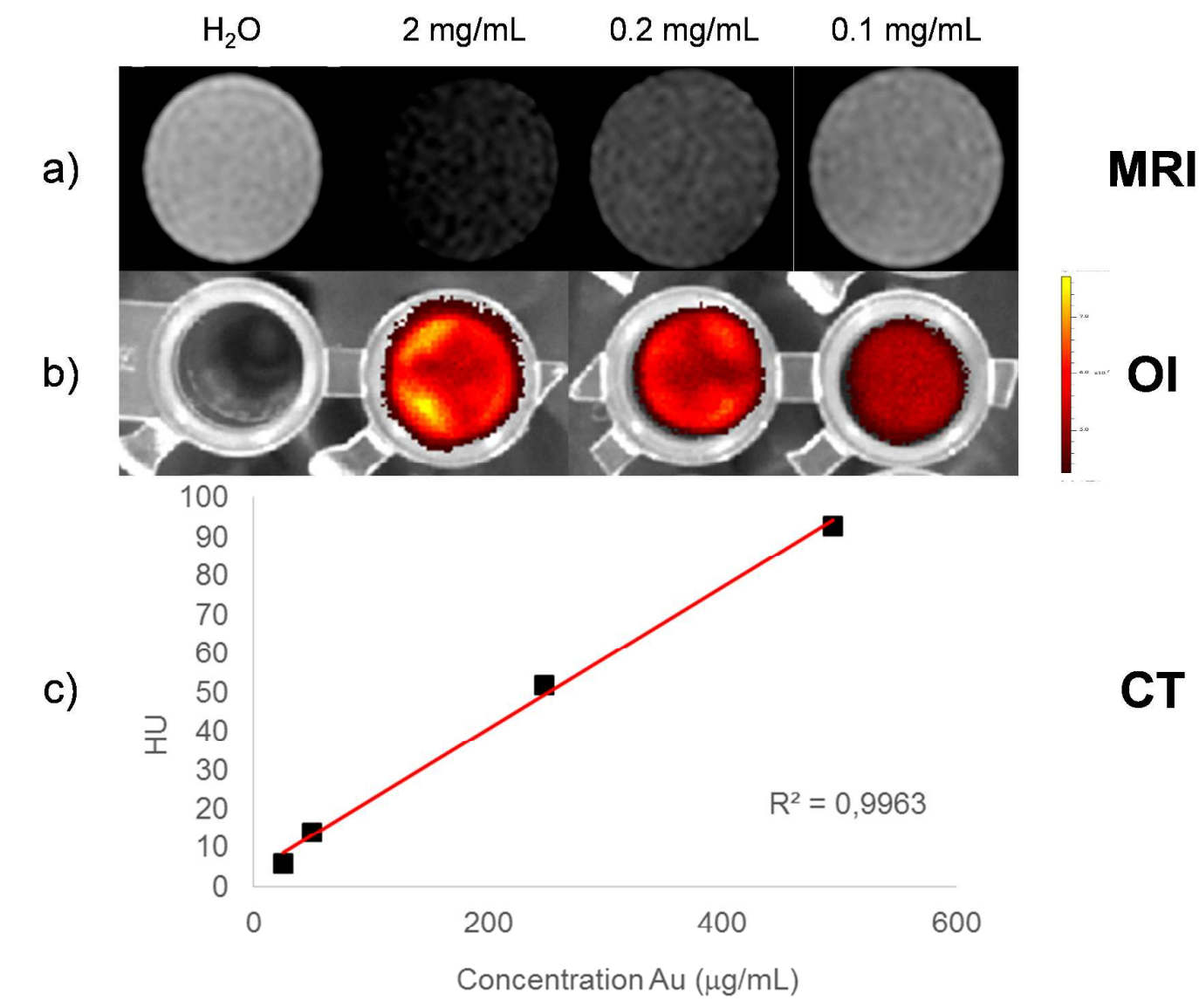

Figure 5. Contrast imaging phantoms for RAM. $\mathrm{T}_{2}$-weighted MRI (a); fluorescence emission spectrum after excitation with Cy 5.5 ex/em filter (b); Standard curve of CT values expressed as Hounsfield units(HU) vs different concentrations of RAM (c). 
As expected, a negative black contrast was promoted in a concentration-dependent manner (Figure 5a). On continuation, the performances of multimodal Janus nanoparticles were assessed in fluorescence imaging by the same concentration-dependent calibration assay previously described for MRI. The scope was the evaluation of fluorescence dye's properties in presence of AuNPs and specially to figure out whether AuNPs quenched the emission fluorescence spectra of fluorophore or not. As it is shown in Figure 5b, the larger nanoprobe concentration the stronger fluorescence intensity. This concentration-dependent fluorescence imaging demonstrated that the fluorescence of RAM was not completely quenched in situ by AuNPs, showing good fluorescence emission properties hence being a good candidate also for optical tracking. Finally, a good CT molecular probe must present a high X-ray coefficient of attenuation..$^{25,26}$ Therefore, to evaluate this property, the HU experimental values of candidate nanoparticles at different concentrations were tested and calculated using a micro PET/CT scanning system. As reported in Figure 5c, the obtained results retrieved a linear relationship $\left(\mathrm{R}^{2}=0.9963\right)$ with good correlation between RAM concentration and HU suggesting that RAM is also an ideal candidate for a positive CT imaging.

\section{In vitro cytotoxicity assessment of RAM.}

Prior to the in vivo application of Janus nanoparticles as multimodal imaging probes, the in vitro cytocompatibility of RAM has been evaluated in order to assess the safety of Janus platform. Therefore, HEK293, HepG2, RAW 264.7, adult fibroblasts (MAF) and fibrosarcome (MF) mouse cells were incubated with Janus RAM at different concentrations (within $35-350 \mu \mathrm{g} / \mathrm{mL}$ range) for $24 \mathrm{~h}$. After that, an MTT colorimetric assay was carried out to assess the cell viability (Figure S6). The achieved results revealed that these nanoparticles were nontoxic over the applied concentration range. In fact, differences in cytotoxicity were not significant (Figure S6). Consequently, as safe concentration, we decided to keep within this range the RAM dose to be administered for subsequent in vivo studies. 


\section{In vivo tumor-targeted multimodal molecular imaging using RAM.}

After checking in vitro the good MRI/CT/OI contrast properties and cytocompatibility of RAMs, we finally assessed the multimodal imaging properties of Janus nanoparticles for in vivo biomedical application. Toward this scope, 3-methylcholanthrene-induced fibrosarcomabearing mice were used. In order to finely analyze the interactions of nanocarriers with tumor, we started analyzing the tumor mice models treated with targeted Janus particle by MRI imaging (Figure 6 and S7).

After tail intravenous injection of RAM, MRI images were acquired at different time postinjection, being $2 \mathrm{~h}$ p.i. the time point that retrieved maximum signal (Figure S7a and S7b). In fact, after $2 \mathrm{~h}$ accumulation, a clear hypointense signal delimiting the tumor area was observed with $\mathrm{T}_{2}$-weighted MRI imaging (Figure 6a $v_{s} 6 \mathrm{~b}$ ).
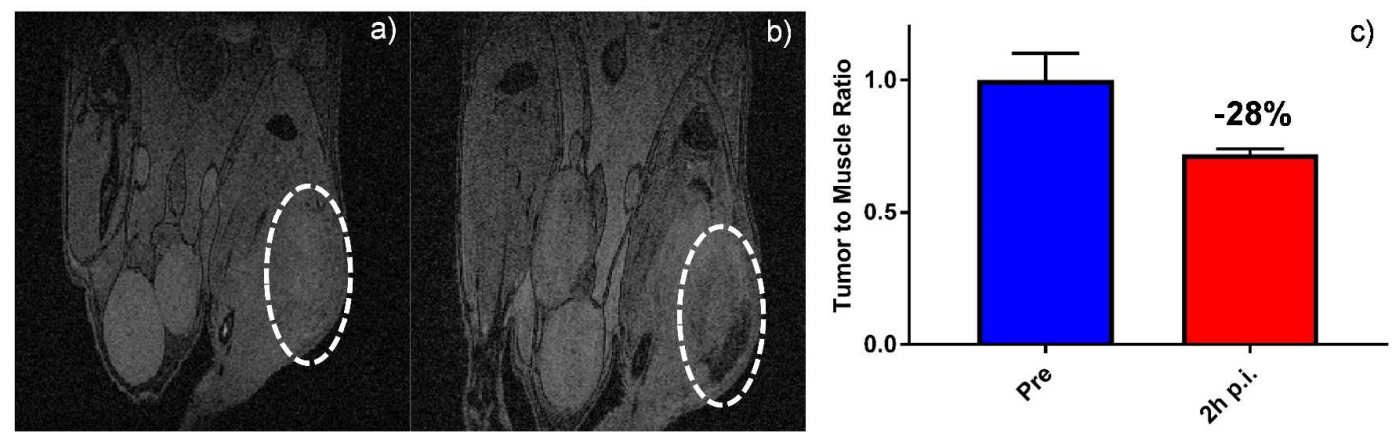

Figure 6. Coronal view for $\mathrm{T}_{2}$-weighted gradient echo 3D MRI of the tumor area in a mouse previously to the injection of targeted Janus RAM (a), $2 \mathrm{~h}$ post-injection (b) and relative MRI mean hypointensity (related to a ROI located in the leg muscle)(c).

These results were also confirmed by signal quantification. The tumor-to-muscle relative signal intensity of tumor area $2 \mathrm{~h}$ post injection decayed in $28 \%$ when compared with basal imaging (Figure 6c and Figure S7b). The RAM distribution was heterogeneous showing a higher uptake in the tumor periphery, as expected by the cRGD peptide mediated neoangiogenesis targeting and in agreement with the fluorescence imaging previously carried out. 
The results achieved by MRI imaging were also confirmed by optical imaging modality. In fact, as reported in Figure 7a, the tumor area clearly showed an intense fluorescence signal due to the presence of targeted Janus particles after $2 \mathrm{~h}$ accumulation following the intravenous injection of RAM.

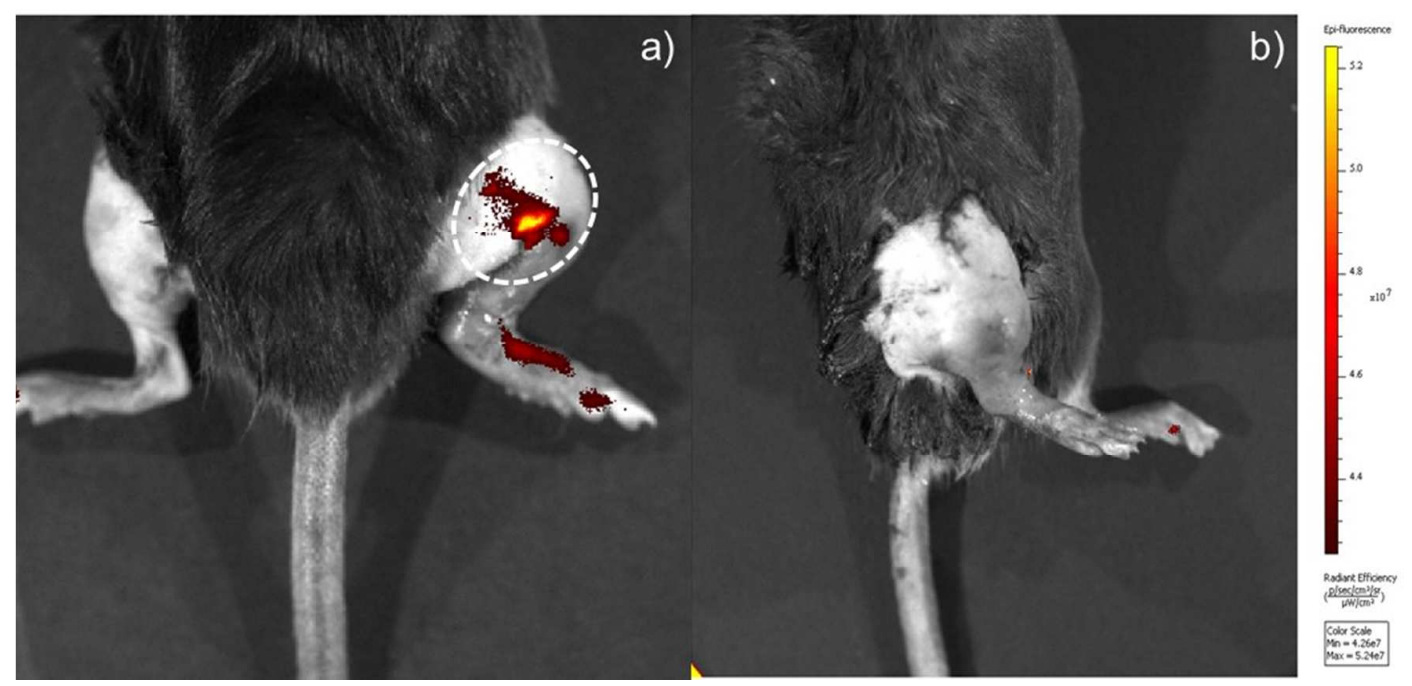

Figure 7. Fluorescence imaging $2 \mathrm{~h}$ p.i of RAM (a) and after blocking experiment by injection of soluble cRGD before RAM administration (b).

This result indicated that, also in vivo, RAM retained sufficient fluorescence to overcame the possible quenching caused by $\mathrm{Au}$ nanoparticles and tissue attenuation, ${ }^{52}$ hence confirming its usefulness for in vivo fluorescence imaging tracking. To confirm these promising results, a control 'blocking' experiment was performed in order to demonstrate that the fluorescence was directly related to the accumulation of intact Janus particles, mediated by the cRGD functionality as the driving force for their site-targeting. In this experiment, freely soluble cRGD peptide ( $1 \mathrm{mg}$ ) was injected previously to the nanoparticles (5 minutes) blocking most of the $\alpha v \beta_{3}$ integrin binding sites and reducing or eliminating the accumulation of the cRGDtargeted RAM nanoparticles. The comparison of Figure $7 \mathrm{~b} v s$ a clearly shows that, after blocking, no fluorescence signal was detected in the tumor region. These results confirm the cRGD-driven tumor accumulation of the Janus particles instead of the passive diffusion effect. The results retrieved by 'blocking' experiment tracked by fluorescence imaging were 
also confirmed by MRI (Figure S7c). In fact, even by this imaging modality, the presence of contrast agent was not appreciated.

Finally, based on the potential of AuNPs as contrast agent for X-Rays, we analyzed the treated tumor-bearing mice by Computed Tomography (Figure 8).
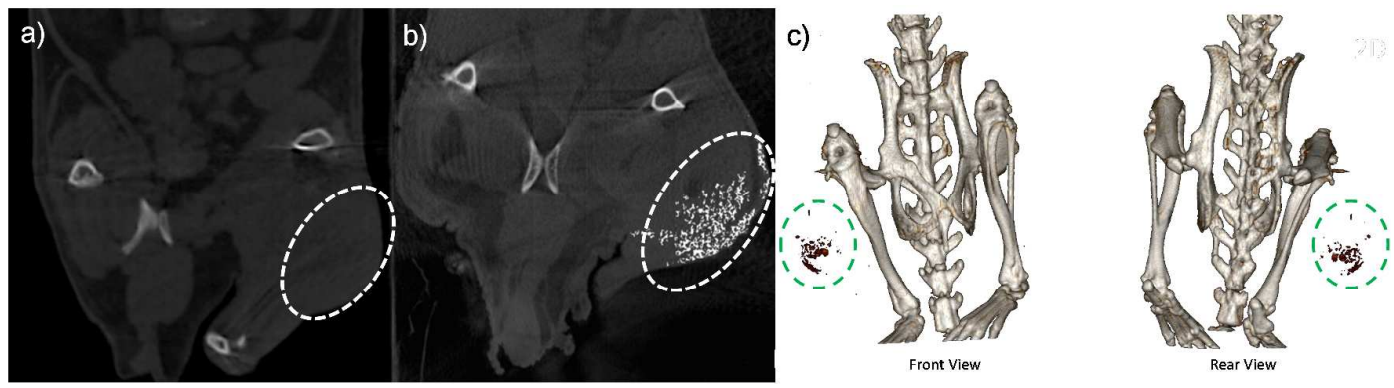

Figure 8. Coronal CT image of the tumor area in mice model before injecting targeted Janus RAM (a), $2 \mathrm{~h}$ post-injection of targeted Janus RAM (b) and 3D stereo images (c).

After $2 \mathrm{~h}$ post-injection, the tumor region showed a clear enhancement of CT signal of more than 2 folds in term of $\mathrm{HU}\left(\mathrm{HU}_{\text {Pre }}: 68 v s \mathrm{HU}_{2 \mathrm{~h} \mathrm{p.i.}}:\right.$ 147; Figure $8 \mathrm{a} v s \mathrm{~b}$ ), indicating that the targeted Janus nanoprobes are also useful as molecular agent for in vivo CT imaging.

Finally, once demonstrated the multimodal molecular imaging ability, we also investigated the biodistribution pattern of RAM after intravenous injection. The accumulation of Janus nanocarriers in the major organs (heart, liver, spleen, lung, kidney and tumor) was examined and quantified by fluorescence imaging after organ excision (Figure S8). The comparison of treated $v s$ not treated tumor mice models clearly indicates that these nanocarriers accumulate quite completely in liver and tumor with a very slight distribution in kidney (Figure S8). No fluorescent signal was appreciated in the other organs. Thereafter, this biodistribution pattern also indicates the hepatobiliary clearance as the preferential metabolic system for these nanocarriers.

\section{Conclusions}


In sum, we have described the preparation of a novel Janus nanoplatform enabling the targeted multimodal molecular in vivo imaging of tumor. In particular, we have reported the preparation of two-faced Janus-type nanoparticles having AuNPs and magnetic NP@MS core@shell at the opposite sides. By means of their different chemical nature, each side has enabled its orthogonal modification by applying selectively the silane (for cRGD tumortargeting peptide) or the sulfur-gold (for NIR probe) chemistries. After deep characterization, the multimodal molecular imaging potential of the as prepared Janus nanoparticles has been successfully evaluated in vitro as well as in vivo. In more details, the Janus nanoplatform enabled the in vivo targeted bioimaging of fibrosarcoma tumor in mice models by MRI (because of the magnetite core) and CT (due to AuNPs) imaging modalities. Furthermore, due to the presence of fluorescence probe, also the optical tracking of targeted Janus nanoparticles has been successfully achieved.

Hence, a novel flexible anisotropic nanoplatform for enhanced multimodal molecular imaging and characterized by high spatial resolution, high sensitivity, deep tissue penetration and easy operational features has been achieved. Considering that I) we have demonstrated already the therapeutic potential of this Janus nanoplatform in controlled drug delivery for cancer treatment (stimuli-responsive doxorubicin release) and II) the presence of both iron as well as gold NPs can enable complementary therapeutic strategies (magnetic hyperthermia and photothermal therapy, respectively), we envision that this overall strategy (the sum of therapeutic and diagnostic potentials) can be successfully applied to generate a novel powerful stimuli-responsive smart nanocarrier useful for advanced theranosis of cancer in particular or in personalized medicine in general.

\section{Acknowledgments}


The CNIC is supported by the Spanish Ministerio de Ciencia, Innovación y Universidades and the Pro-CNIC Foundation and is a Severo Ochoa Center of Excellence (SEV-2015-0505). M.F. acknowledges Ministerio de Economia y Competitividad for the research grant no. SAF2014-59118-JIN co-funded by Fondo Europeo de Desarrollo Regional (FEDER) and COST Action CA1520: 'European Network on NMR Relaxometry-EURELAX'. M.F. also acknowledges the Comunidad Autonoma de Madrid for research project num. 2017-T1/BIO4992 ('Atracción de Talento’ Action) also cofunded by Universidad Complutense de Madrid. Financial support from the MEyC Projects CTQ2014-58989-P, CTQ2017-87954-P, SAF2017-844494-C2-1R and CTQ2015-71936-REDT and the Comunidad de Madrid Projects: Programme NANOAVANSENS (Project S2013/MIT-3029) and the I+D collaborative Programme in Biomedicine NIETO-CM (Project reference B2017-BMD3731) are gratefully acknowledged. The authors would like to thank Ms Ana Victoria Lechuga Vieco for her help in preparing animal models and Izaskun Bilbao for the assistance in images acquisition.

\section{ASSOCIATED CONTENT}

\section{Supporting Information}

The Supporting Information is available free of charge on the ACS Publications website at DOI: $10.1021 /$ acsami.xxxxxxx.

TEM micrographs of Mag 320, Mag 320@MS and Au-Mag 320@MS, size distribution analysis of Mag 320; HRTEM micrograph of Mag 320, Fluorescence spectra of Janus AuMag 320@MS before and after modification with Alexa Fluor 647; FTIR spectra of Mag 320, Mag 320@MS, Au-Mag 320@MS and RAM nanoparticles; Cytotoxicity assay of RAM using different cell lines at various concentrations; Mouse MRI images acquired at different time 
post-injection of RAM, and after blocking experiment with soluble cRGD; Biodistribution of RAM in mice; DLS and ICP-AES values from the RAM nanoparticles analysis.

\section{Notes}

The authors declare no competing financial interest. 


\section{References}

(1) Chan, I. S.; Ginsburg, G. S. Personalized Medicine: Progress and Promise. Annu. Rev. Genomics Hum. Genet. 2011, 12, 217-244.

(2) Mura, S.; Couvreur, P. Nanotheranostics for Personalized Medicine. Adv. Drug Deliv. Rev. 2012, 64, 1394-1416.

(3) Rai, P.; Mallidi, S.; Zheng, X.; Rahmanzadeh, R.; Mir, Y.; Elrington, S.; Khurshid, A.; Hasan, T. Development and Applications of Photo-triggered Theranostic Agents. Adv. Drug Deliv. Rev. 2010, 62, 1094-1124.

(4) Lim, E.-K.; Kim, T.; Paik, S.; Haam, S.; Huh, Y.-M.; Lee, K. Nanomaterials for Theranostics: Recent Advances and Future Challenges. Chem. Rev. 2015, 115, 327-394.

(5) Janib, S. M.; Moses, A. S.; MacKay, J. A. Imaging and Drug Delivery using Theranostic Nanoparticles. Adv. Drug Deliv. Rev. 2010, 62, 1052-1063.

(6) Wei, R.; Xi, W.; Wang, H.; Liu J.; Mayr, T.; Shia, L.; Sun, L. In Situ Crystal Growth of Gold Nanocrystals on Upconversion Nanoparticles for Synergistic Chemo-Photothermal Therapy. Nanoscale, 2017, 9, 12885-12896.

(7) Wei, R.; Wei, Z.; Sun, L.; Ahang, J. Z.; Liu, J.; Ge, X.; Shi, L. Nile Red DerivativeModified Nanostructure for Upconversion Luminescence Sensing and Intracellular Detection of $\mathrm{Fe}^{3+}$ and MR Imaging. ACS Appl. Mater. Interfaces, 2016, 8, 400-410.

(8) Sun, L.; Wei, R.; Feng, J.; Zhang, H. Tailored Lanthanide-Doped Upconversion Nanoparticles and Their Promising Bioapplication Prospects. Coord. Chem. Rev., 2018, 364, $10-32$.

(9) Erami, R. S.; Ovejero, K.; Meghdadi, S.; Filice M.; Amirnasr, M.; Rodríguez-Diéguez, A.;

De La Orden, M. U.; Gómez-Ruiz, S. Applications of Nanomaterials Based on Magnetite and 
Mesoporous Silica on the Selective Detection of Zinc Ion in Live Cell Imaging. Nanomaterials, 2018, 8, 434-460.

(10) Ganta, S.; Devalapally, H.; Shahiwala, A.; Amiji, M. A review of Stimuli-Responsive Nanocarriers for Drug and Gene Delivery. J. Control. Release 2008, 126, 187-204.

(11) Roh, K. H.; Martin, D. C.; Lahann, J. Biphasic Janus Particles with Nanoscale Anisotropy, Nat. Mater. 2005, 4, 759-763.

(12) Binks, B.; Fletcher, P. Particles Adsorbed at the Oil-Water Interface: a Theoretical Comparison between Spheres of Uniform Wettability and “Janus" Particles, Langmuir 2001, $17,4708-4710$.

(13) Tran, L. T. C.; Lesieur, S.; Faivre, V. Janus Nanoparticles: Materials, Preparation and Recent Advances in Drug Delivery, Expert Opin. Drug Deliv. 2014, 11, 1061-1074.

(14) Shao, D.; Li, J.; Zheng, X.; Pan, Y.; Wang, Z.; Zhang, M.; Chen, Q.-X.; Dong, W.-F.; Chen, L. Janus "Nano-Bullets" for Magnetic Targeting Liver Cancer Chemotherapy, Biomaterials 2016, 100, 118-133.

(15) Díez, P.; Sánchez, A.; Gamella, M.; Martínez-Ruíz, P.; Aznar, E.; de la Torre, C.; Murguía, J. R.; Martínez-Máñez, R.; Villalonga, R.; Pingarrón. J. M. Toward the Design of Smart Delivery Systems Controlled by Integrated Enzyme-Based Biocomputing Ensembles. J. Am. Chem. Soc. 2014, 136, 9116-9123.

(16) Ling, D.; Hackett, M. J.; Hyeon, T. Cancer Imaging: Lighting up Tumours. Nat Mater 2014, 13, 122-124.

(17) Cormode, D. P.; Jarzyna, P. A.; Mulder, W. J.; Fayad, Z. A. Modified Natural Nanoparticles as Contrast Agents for Medical Imaging. Adv. Drug Deliv. Rev. 2010, 62, 329338. 
(18) Wysocki, L. M.; Lavis, L. D. Advances in the Chemistry of Small Molecule Fluorescent Probes. Curr. Opin. Chem. Biol. 2011, 15, 752-759.

(19) Baker, M. Whole-Animal Imaging: the Whole Picture. Nature 2010, 463, 977-980.

(20) Sailor, M. J.; Park, J. H. Hybrid Nanoparticles for Detection and Treatment of Cancer. Adv. Mater. 2012, 24, 3779-3802.

(21) Lee, D. E.; Koo, H.; Sun, I. C.; Ryu, J. H.; Kim, K.; Kwon, I. C. Multifunctional Nanoparticles for Multimodal Imaging and Theragnosis. Chem. Soc. Rev. 2012, 41, 26562672.

(22) Marciello, M.; Pellico, J.; Fernandez-Barahona, I.; Herranz, F.; Ruiz-Cabello, J.; Filice, M. Recent Advances in the Preparation and Application of Multifunctional Iron Oxide and Liposome-based Nanosystems for Multimodal Diagnosis and Therapy. Interface Focus 2016, 6, 20160055.

(23) Cheng, Z.; Al Zaki, A.; Hui, J. Z.; Muzykantov, V. R.; Tsourkas, A. Multifunctional Nanoparticles: Cost versus Benefit of Adding Targeting and Imaging Capabilities. Science 2012, 338, 903-910.

(24) Huang, Y.; He, S.; Cao, W.; Cai, K.; Liang, X. J. Biomedical Nanomaterials for ImagingGuided Cancer Therapy. Nanoscale 2012, 4, 6135-6149.

(25) Lee, N.; Choi, S. H.; Hyeon, T. Nano-sized CT Contrast Agents. Adv. Mater. 2013, 25, 641-660.

(26) Lusic, H.; Grinstaff, M. W. X-Ray-Computed Tomography Contrast Agents. Chem. Rev. 2012, 113, 1641-1666. 
(27) Cole, L. E.; Ross, R. D.; Tilley, J. M.; Vargo-Gogola, T.; Roeder, R. K. Gold Nanoparticles as Contrast Agents in X-Ray Imaging and Computed Tomography. Nanomedicine 2015, 10, 321-341.

(28) Qin, W.; Ding, D.; Liu, J. Z.; Yuan, W. Z.; Hu, Y.; Liu, B.; Tang, B. Z. Biocompatible Nanoparticles with Aggregation-Induced Emission Characteristics as Far-Red/Near-Infrared Fluorescent Bioprobes for in vitro and in vivo Imaging Applications. Adv. Funct. Mater. 2012, $22,771-779$.

(29) Byrne, W. L.; DeLille, A.; Kuo, C.; de Jong, J. S.; van Dam, G. M.; Francis, K. P.; Tangney, M. Use of Optical Imaging to Progress Novel Therapeutics to the Clinic. J. Control Release 2013, 172, 523-534.

(30) Eckert, M. A.; Vu, P. Q.; Zhang, K.; Kang, D.; Ali, M. M.; Xu, C.; Zhao, W. Novel Molecular and Nanosensors for in vivo Sensing. Theranostics 2013, 3, 583-594.

(31) Shin, T. H.; Choi, Y.; Kim, S.; Cheon, J. Recent Advances in Magnetic Nanoparticlebased Multi-Modal Imaging. Chem. Soc. Rev. 2015, 44, 4501-4516.

(32) Liong, M.; Lu, J.; Kovochich, M.; Xia, T.; Ruehm, S. G.; Nel, A. E.; Tamanoi, F.; Zink, J. I. Multifunctional Inorganic Nanoparticles for Imaging, Targeting, and Drug Delivery. ACS Nano 2008, 2, 889-896.

(33) Frens, G. Controlled Nucleation for the Regulation of the Particle Size in Monodisperse Gold Suspensions. Nature 1973, 241, 20-22.

(34) Villalonga, R.; Díez, P.; Sánchez, A.; Aznar, E.; Martínez-Máñez, R.; Pingarrón, J. M. Enzyme-Controlled Sensing-Actuating Nanomachine based on Janus Au-Mesoporous Silica Nanoparticles. Chem. Eur. J. 2013, 19, 7889-7894.

(35) DiGiovanni, J. Multistage Carcinogenesis in Mouse Skin. Pharmac. Ther. 1992, 54, 63128. 
(36) Li, W.; Lv, B.; Xu, Y. Sub-30 nm $\mathrm{Fe}_{3} \mathrm{O}_{4}$ and c- $\mathrm{Fe}_{2} \mathrm{O}_{3}$ Octahedral Particles: Preparation and Microwave Absorption Properties. J. Nanopart. Res. 2013, 15, 2114-2123.

(37) Martin, M.; Salazar, P.; Villalonga, R.; Campuzano, S.; Pingarrón, J. M.; González-Mora, J. L. Preparation of Core-Shell $\mathrm{Fe}_{3} \mathrm{O}_{4} @$ @oly(Dopamine) Magnetic Nanoparticles for Biosensor Construction. J. Mater. Chem. B 2014, 2, 739-746.

(38) Chen, Y.; Chen, A.; Qui, J. Polystyrene Core-Silica Shell Composite Particles: Effect of Mesoporous Shell Structures on Oxide CMP and Mechanical Stability. RSC Adv. 2017, 7, 6548-6558.

(39) Zhao, Y. X.; Gao, C. G.; Li, Y. X.; Zhang, T. M. Wormhole-like Mesoporous Silica Templated by Double-Chained Cationic Surfactant. Micropor. Mesopor. Mat. 2009, 124, 4244.

(40) Silva, V. A. J.; Andrade, P.L.; Silva, M. P. C.; Bustamante, A.; Luis De Los Santos Valladares, D.; Albino Aguiar, J. Synthesis and Characterization of $\mathrm{Fe}_{3} \mathrm{O}_{4}$ Nanoparticles Coated with Fucan Polysaccharides. J. Magn. Magn. Mater. 2013, 343, 138-143.

(41) Thommes, M.; Kaneko, K.; Neimark, A. V.; Olivier, J. P.; Rodriguez-Reinoso, F.; Rouquerol, J.; Sing, K. S. W. Physisorption of Gases, with Special Reference to the Evaluation of Surface Area and Pore Size Distribution (IUPAC Technical Report). Pure Appl. Chem. 2015, 87, 1051-1069.

(42) Perez De Berti, I. O.; Cagnoli, M. V.; Pecchi, G.; Alessandrini, J. L.; Stewart, S. J.; Bengoa, J. F.; Marchetti, S. G. Alternative Low-Cost Approach to the Synthesis of Magnetic Iron Oxide Nanoparticles by Thermal Decomposition of Organic Precursors. Nanotechnol. 2013, 24, 175601-175612. 
(43) Daniel, M. C.; Astruc, D. Gold Nanoparticles: Assembly, Supramolecular Chemistry, Quantum-Size-Related Properties, and Applications toward Biology, Catalysis, and Nanotechnology. Chem. Rev. 2004, 104, 293-346

(44) Gaertner, F. C.; Kessler, H.; Wester, H.-J.; Schwaiger, M.; Beer, A. J. Radiolabelled RGD Peptides for Imaging and Therapy. Eur. J. Nucl. Med. Mol. Imaging 2012, 39, 126-138.

(45) Liu, Z.; Peng, R. Inorganic Nanomaterials for Tumor Angiogenesis Imaging. Eur. J. Nucl. Med. Mol. Imaging 2010, 37, S147-63.40

(46) Barth, A. The Infrared Absorption of Amino Acid Side Chains. Prog. Biophys. Mol. Biol. 2000, 74, 141-173.

(47) Teng, Z.; Li, J.; Yan, F.; Zhao, R.; Yang, W. Highly Magnetizable Superparamagnetic Iron Oxide Nanoparticles Embedded Mesoporous Silica Spheres and their Application for Efficient Recovery of DNA from Agarose Gel. J. Mater. Chem. 2009, 19, 1811-1815.

(48) Laurent, S.; Forge, D.; Port, M.; Roch, A.; Robic, C.; Vander Elst, L.; Muller, R. N. Magnetic Iron Oxide Nanoparticles: Synthesis, Stabilization, Vectorization, Physicochemical Characterizations, and Biological Applications. Chem. Rev. 2008, 108, 2064-2110.

(49) Zahraei, M.; Marciello, M.; Lazaro-Carrillo, A.; Villanueva. A.; Herranz, F.; Talelli, M.; Costo, R.; Monshi, A.; Shahbazi-Gahrouei, D.; Amirnasr, M.; Behdadfar, B.; Morales, M. P. Versatile Theranostics Agents Designed by Coating Ferrite Nanoparticles with Biocompatible Polymers. Nanotechnol. 2016, 27, 255702.

(50) Lee, J. H.; Huh, Y. M.; Jun, Y. W.; Seo, J. W.; Jang, J. T.; Song, H. T.; Kim, S.; Cho, E. J.; Yoon, H. G.; Suh, J. S.; Cheon, J. Artificially engineered magnetic nanoparticles for ultrasensitive molecular imaging. Nat Med. 2007, 13, 95-99.

(51) Seo, W. S.; Lee, J. H.; Sun, X.; Suzuki, Y.; Mann, D.; Liu, Z.; Terashima, M.; Yang, P. C.; McConnell, M. V.; Nishimura D. G.; Dai, H. FeCo/Graphitic-Shell Nanocrystals as 
Advanced Magnetic-Resonance-Imaging and Near-Infrared Agents. Nature Mat. 2006, 5, 971-976.

(52) Li, F.; Pei, H.; Wang, L. H.; Lu, J. X.; Gao, J. M.; Jiang, B. W.; Zhao, X. C.; Fan, C. H. Nanomaterial-based Fluorescent DNA Analysis: a Comparative Study of the Quenching Effects of Graphene Oxide, Carbon Nanotubes, and Gold Nanoparticles. Adv. Funct. Mater. 2013, 23, 4140-4148. 


\section{Graphical abstract}

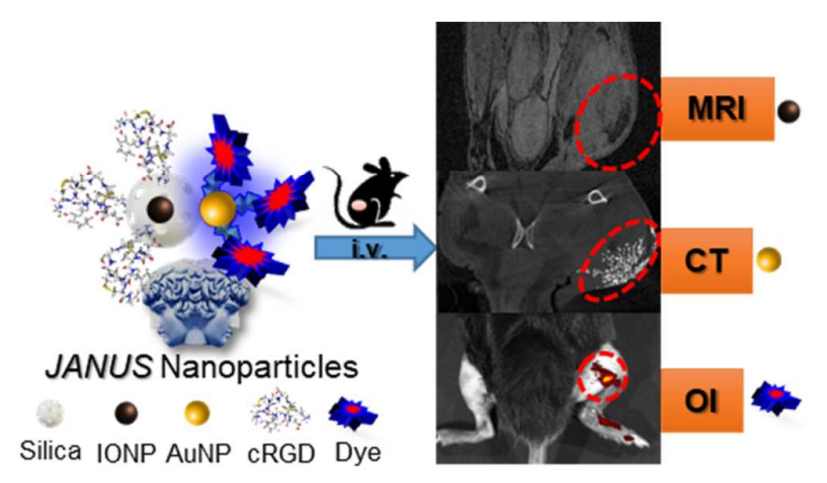

15

16 